\title{
Pattern of UTI in Chronic Kidney Disease: Experience from A Tertiary Care Hospital, Bangladesh
}

\author{
Marina Arjumand ${ }^{1 *}$ \\ Golam Mohammad Tayeb Ali ${ }^{2}$ \\ Pradip Kumar Dutta ${ }^{1}$ \\ Md. Habib Hassan ${ }^{3}$ \\ Kazi Md. Abrar Hasan ${ }^{3}$ \\ Biplob Kumar Barua'
}

'Department of Nephrology Chittagong Medical College Chattogram, Bangladesh.

${ }^{2}$ Department of Pediatrics Rangamati Medical College Rangamati, Bangladesh.

${ }^{3}$ Department of Medicine Chittagong Medical College Chattogram, Bangladesh.

\section{*Correspondence to:}

\section{Dr. Marina Arjumand}

Consultant

Department of Nephrology

Chittagong Medical College

Chattogram, Bangladesh.

Mobile : +88 01717224422

Email : drmarinaarjumand@gmail.com

Date of Submission : 02.11 .2020

Date of Acceptance : 20.12 .2021

www.banglajol.info/index.php/CMOSHMCJ

\begin{abstract}
Background: Urinary Tract Infection (UTI) is common and higher in prevalence in patients with Chronic Kidney Disease (CKD). To find out the frequency and clinicobacteriological pattern of UTI in CKD patients.
\end{abstract}

Materials and methods: A cross-sectional study was done on 1000 of CKD patients attending Nephrology Department of Chattogram Medical College Hospital (CMCH) a tertiary level hospital in Bangladesh during January to December, 2017. Cleancatch midstream urine samples were collected from study populations irrespective of symptoms. Macroscopic and microscopic examinations were done. Urine samples were then inoculated in Blood agar and Mac'Conkey agar and incubated aerobically at $37{ }^{\circ} \mathrm{C}$ for 18-24 hours. Microscopic observations and conventional biochemical tests were done to identify the isolated organisms. Significant growth of organisms in urine was defined as UTI. The isolated organisms were tested for antimicrobial susceptibility by using modified Kirby Bauer technique.

Results: The prevalence of UTI was $61.8 \%$. with significant asymptomatic UTI for 28\%. Gram-negative organisms were most frequent 564 (93\%) with predominant Escherichia coli 399 (64\%) followed by Klebsiella 113(18\%) and Pseudomonas 39(6\%). Among Gram positive isolates, Staphylococcus (6\%) was the dominant organism isolated. Regarding antibiotic susceptibility, Gram-negative microorganisms were found to be most sensitive to carbapenem and amikacin whereas Gram-positive microorganisms were most sensitive towards carbapenem, colistin and linezolid.

Conclusion: In CKD patients, asymptomatic UTI was detected significantly. E.coli was found to be predominant organisms and resistant towards most of the studied common antibiotics.

Key words: Bacteriuria; Asymptomatic UTI; Chronic kidney disease; Antibiotic susceptibility.

\section{INTRODUCTION}

Chronic Kidney Disease (CKD) is now a global problem ${ }^{1}$. Infection is one of the detrimental factors for greater morbidity and mortality in these patients ${ }^{2}$. UTIs are one of the most common infections in CKD, accounting to $35 \%$ of nosocomial infections $^{3,4}$. A deterioration in renal function correlates with disturbed specific and non-specific host defenses ${ }^{5}$. A change in the composition of urine, oliguria, anuria and the resultant changes in $\mathrm{pH}$, osmolality and urinary urea have effect on UTIs. Various uraemic toxins inhibit antimicrobial activity of granulocytes, macrophages and other defense reactions. These may contribute to develop UTI in patients with renal diseases ${ }^{6,7}$. Due to structural and functional defect, the incidence of UTI in CKD is higher compared to others ${ }^{8}$. The risk might be increased by disease factors and management of comorbidities ${ }^{9}$. In the patients with the chronic renal insufficiency, urine drug concentration may be too low to eradicate organisms completely. Resultant treatment failure and compromised host immune system increases the vulnerability to infections ${ }^{10}$. 
The patients with renal insufficiency, UTI may be asymptomatic or with non-specific symptoms and early accurate diagnosis is not always easy. Delayed treatment may contribute to further loss of renal function. So surveillance of urinary pathogens in a hospital is important for both clinical and microbiological. The data of UTI in CKD patients yet demands more informations. This study is aimed to find out the prevalence of UTI in CKD irrespective of symptoms and to identify the bacterial pathogens from urine samples and their sensitivity pattern.

\section{MATERIALS AND METHODS}

This cross-sectional study was conducted on patients of CKD admitted to Nephrology Department of CMCH during January to December 2017. CKD patients of both sex aged $\geq 18$ years with or without dialysis were included consecutively irrespective of symptoms of UTI. CKD patients with pyuria getting antibiotic for more than 48 hours before admission or with anuria and who did not provide written consent were excluded. A preformed standard case record form was used for data collection. A midstream clean-catch urine sample at least $>20 \mathrm{ml}$ was collected in the clean, sterile, dry container by standard procedure. Examination and culture of urine: Urine samples were tested by using multiple reagent strips and microscopic urinalysis. A urine dipstick consists of chemically treated paper, which displays different colors indicating the presence of sugar, protein, specific gravity and $\mathrm{pH}$. The cut-off value for pyuria was determined $>10$ pus cell/HPF by cell count method. All samples were inoculated on Blood agar and Mac-Conkey agar by the semiquantatitive culture technique using a standard wire loop and incubated at $37^{\circ} \mathrm{C}$ for $24-48$ hours in an incubator. The approximate number of colonies and the number of bacteria was counted. Bacteriuria was defined as any micro-organisms growth of $\geq 10^{5} \mathrm{CFU} / \mathrm{ml}$ or $\geq 10^{3} \mathrm{CFU} / \mathrm{ml}$ in symptomatic patients. Asymptomatic Bacteriuria (ASBU) was defined as the presence of at least $10^{5}$ colony-forming units $(\mathrm{CFU} / \mathrm{ml})$ of bacterial species in clean-voided midstream urine sample without symptoms of a UTI ${ }^{11,12}$. The isolated organisms were identified by standard biochemical method which involves morphological appearance of the colonies, staining reactions and biochemical properties ${ }^{13}$. Antimicrobial susceptibility of isolates was tested by Kirby-Bauer disk diffusion method using Mueller-Hinton agar. Results were read according to the National Committee for Clinical Laboratory Standards guidelines (NCCLS) ${ }^{14,15}$. Ethical Committee approval ( Memo No. CMC/PG/2017/315) was duly obtained from the Institutional Review Board of Chittagong Medical College Hospital, Chattogram prior commencement of the study.

Data were collected by interview \& recording reports of laboratory investigations. All the collected data were checked and compiled and then tabulated. The data were entered into SPSS for windows 20( SPSS in C Chicago, IL,USA). All data were evaluated by using Chi-square test for categorical variables and ttest for continuous variables. The results were presented in Tables and Figures. Mean for numerical variables and percentage for categorical variables was used as statistical term in this study. AOR and multiple regression analysis was done for prediction of risk factors. Statistical significance was set at $\mathrm{p}<0.05$ and confidence interval was set at $95 \%$ level.

\section{RESULTS}

This cross-sectional study found 618 subjects $(62 \%)$ with UTI among study population $(\mathrm{n}=1000)$. Distribution of the sociodemographic characteristics showed prevalence of UTI was more in female, poor economic status and in illiterate group with highest $(23 \%)$ in the age group of (51-60) years. Significant UTI was in CKD patients with DM, Stone disease, with Indwelling catheter and on Dialysis (Not shown). The prevalence of UTI was about two third of total patients $(61.8 \%)$ and about one fourth of UTI (28\%) was asymptomatic (Table I). The major organism was gram negative organism and E. coli was the predominant organism followed by klebsiella (Figure 1). E.coli growth is similar in both group but Pseudomonus caused asymptomatic UTI more compared to symptomatic whereas Staphylococcus wass more in symptomatic UTI (Table II). Association between type of organism and symptoms was significant in Chi-square test. The study showed highest sensitivity of E.coli and Klebsiella towards both Meropenem (90\%) and Amikacin $(63 \%, 71 \%)$. Pseudomonus to Ceftazidime (72\%), Meropenem (65\%) (Figure $2 \& 3$ ). Gram positive organism showed most susceptibility towards Pipercillin-Tazobactum and Colistin (100\%), Linezolid (97\%), Clindamycin (93\%) and Carbapenem (90\%).

Table I : Prevalence of UTI and their distribution according to symptoms.

\begin{tabular}{lccrcr} 
Symptom status & \multicolumn{4}{c}{ UTI status } & p-value \\
& \multicolumn{1}{c}{ Present-n (\%) } & \multicolumn{1}{c}{ Absent-n (\%) } & \\
Symptomatic & 458 & $72 \%$ & 297 & $68 \%$ & $.001 *$ \\
Asymptomatic & 160 & $28 \%$ & 85 & $32 \%$ & \\
Total & 618 & $62 \%$ & 382 & $38 \%$ & \\
\hline
\end{tabular}

Table I shows that prevalence of UTI was about two third of total patients and about one fourth of UTI was asymptomatic.

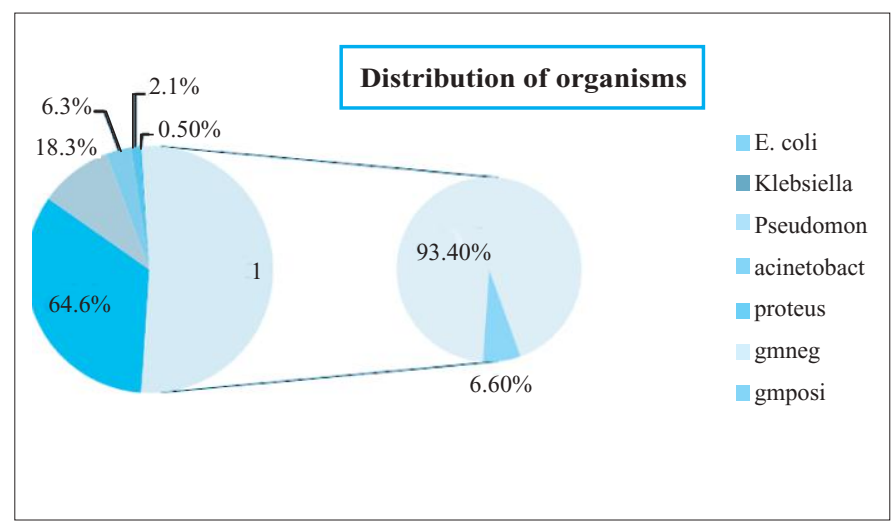

Figure 1: Distribution of uropathogens among the positive UTI samples $(n=618)$.

Figure 1 showed that majority is gram negative organisms and predominant isolate is E.Coli followed by klebsiella and pseudomonas. Staphylococcus is dominant among gram positive organism. 
Table II : Association of bacteriological pattern in symptomatic vs asymptomatic UTI patients.

$\begin{array}{lrrrrrr}\text { UTI patient } & \text { E. Coli } & \text { Klebsiella } & \text { Pseudomonus } & \text { Staphylococcus } & \text { Others } & \text { p Value } \\ \text { Symptomatic } & 296(65 \%) & 87(19 \%) & 22(5 \%) & 35(8 \%) & 17(4 \%) & <0.001 * \\ \text { Asymptomatic } & 103(64 \%) & 26(16 \%) & 17(11 \%) & 3(2 \%) & 9(6 \%) & \end{array}$

Table II showed that Pseudomonus causes asymptomatic UTI more compared to symptomatic whereas Staphylococcus is more frequent in symptomatic UTI

*Association between type of organism and symptoms is significant in Chi-square test.

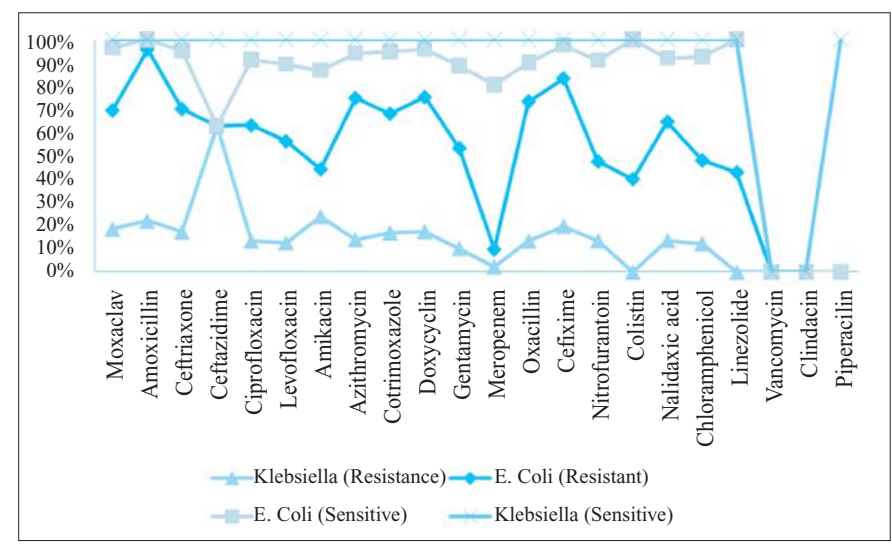

Figure 2 : Susceptibility pattern of E. coli and Klebsiella to different antibiotics.

Figure 2 showed highest sensitivity of E.coli and Klebsiella towards both Meropenem (90\%) and Amikacin (63\%,71\%).

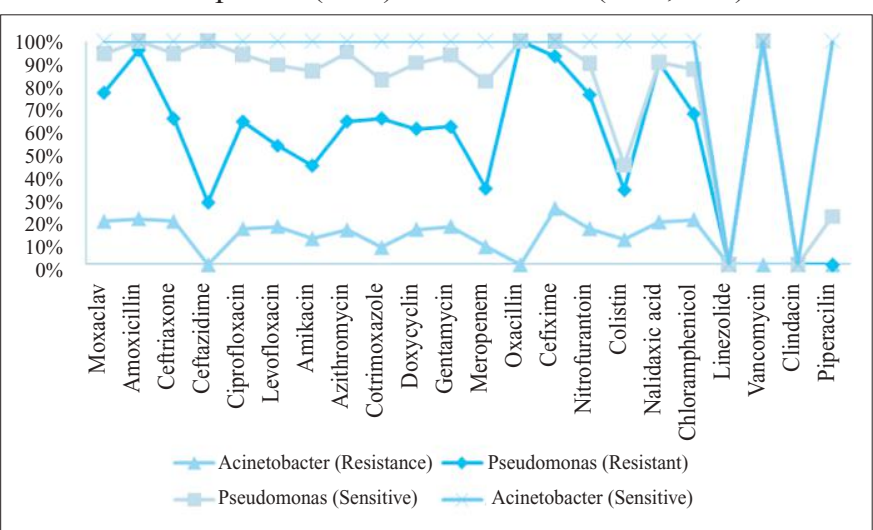

Figure 3 : Susceptibility of antibiotics for Pseudomonus and acinetobacter.

Figure 3 Pseudomonus is mostly sensitive to Ceftazidime (72\%), Meropenem (65\%).

\section{DISCUSSION}

In this study, after adjusting effect sex, DM, dialysis were found significant clinical predictor of UTI in CKD. Maconald et al stated age as risk in CKD which was discrepant with our study ${ }^{16}$. James et al observed a weaker association with age for infection supporting our study ${ }^{17}$. Gatenet et al found no statistical significance for age, sex in evaluation of risk except catheterization ${ }^{18}$. Saitoh et al revealed association of UTI with primary cause with higher frequency in PKD followed by DN and Chronic $\mathrm{GN}^{19}$. Jadav et al found Indwelling Catheter and Richa et al reported stone disease as a predictor of $\mathrm{UTI}^{6,20}$. Dissimilarity of our study may be due small number of sub-group population. Dialysis was found a predictor of UTI in our study which is supported by all other previous studies. Regarding diabetes findings of studies are variable ${ }^{21}$.

In this study, $618(62 \%)$ of participants were found with UTI Similar to Golebiewska et al with $65 \%{ }^{22}$. But the recent study conducted by Jaiswal et al Siddiqui KM and chowdhury Richa et al showed report of $30 \%, 36 \%, 26 \%$ of growth positivity ${ }^{11,13,20}$. The variation may be due to inclusion of specific CKD sub-groups

As CKD stages advances, blunted immune response results in asymptomatic UTI. This was evident in our study with significant asymptomatic UTI (28\%). Prevalence of asymptomatic UTI varies from $9-27 \%$ in various studies ${ }^{23}$. Jaiswal et al also Renko et al suggested that significantly ${ }^{11,24}$. Dysautonomia and defective chemotaxis in diabetic nephropathy and uremic toxins in Hemodialysis (HD) or ESRD patients may cause this ${ }^{7,25,26}$. This was supported with study by Richa et al, Falah et al on HD patients with $p$ value $<0.001^{20,26}$.

In this study, gram negative (gm -ve) bacteria were found more frequent for UTI (93\%) which was similar to study done by Richa et al reported $85 \%$, Yadav et al $97 \%$ and other recent reports ${ }^{11,20,23,26,27}$. Among 7 different isolates E.coli $64.6 \%$ was found to be more predominant and followed by klebsiella $18 \%$, pseudomonas $6.3 \%$, Staphylococcus $6.4 \%$, acinetobacter $2.1 \%$, Proteus $0.5 \%$, Streptococcus $0.2 \%$. This was similar to studies done by Falah et al Yadav et al Ranjana et al HSAIO et al ${ }^{26-29}$.

But some author found staphylococcus one of the commonest organism specially in HD \& transplant patients whereas our study reported $6.6 \%{ }^{30}$. Studies by Suresh found $26.6 \%$, Richa et al $15.4 \%$, venkatesh et al $9.4 \%$, Jamal haider $24.2 \%$, mostly included patients with $\mathrm{HD}^{11,20,23,31}$

Our study found E.coli growth equally responsible for both symptomatic and asymptomatic UTI and Pseudomonas and other Gm -ve bacteria causes asymptomatic UTI more whereas staphylococcus causes symptomatic infection more. This pattern of asymptomatic UTI is similar to other studies but findings by by Richa et al and Suresh et al who found staphylococcus next to E.coli in asymptomatic patients does not support

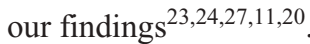

Our study showed highest sensitivity of gram negative organisms towards Meropenem, Amikacin, Colistin, Ceftazidime. Gram positive organism showed most susceptibility towards Pipercillin-Tazobactum, Linezolid and Clindamycin. This sensitivity pattern of our study more or less similar to other studies done in CKD on broad spectrum antibiotics except susceptibility of NFN for gram negative and Oxacilin for gram positive which are almost not a highly active agent in our study ${ }^{26,31,32}$. 
This may explain their prophylactic and over the counter usage in our setup. Most frequently used antibiotics like amoxacilin, cefixime, ciprofloxacin, NFN, ceftriaxone revealed low level of sensitivity to all organisms as a whole. Continuous use of broad spectrum antibiotic during HD period, ESRD and DM patients for any complication resulted these scenario.

\section{CONCLUSION}

In our study female were more prone to UTI and the frequency of asymptomatic UTI was significant in CKD patients. E. coli was the commonest organism which is resistant to most of common antibiotics. As predictor of UTI in CKD patients Sex, DM, and Dialysis were significant after adjustment.

\section{DISCLOSURE}

All the authors declared no conflict of interest.

\section{REFERENCES}

1. R Funfst"uck, U Ott, KG Naber. The interaction of urinary tract infection and renal insufficiency. International Journal of Antimicrobial Agents. 2006;72-77.

2. CO Alebiosu, OO Ayodele, A Abbas et al. Chronic Renal Failure at the Olabisi Onabanjo University Teaching Hospital, Sagamu, Nigeria. African Health Science. 2006 ;6(3):132-138.

3. AS Kolawale, OM Kolawale, YT Kandaki, et al. Prevalence of Urinary Tract Infections (UTI) among patients attending Dahatu Araf Specialists Hospitals Lafia, Nasrawa State, Nigeria. International Journal of Medicine and Medical Sciences . 2009;163-167.

4. N Jha, SK Bapat. A study of sensitivity of resistance of pathogenic microorganisms causing UTI in Kathmandu valley. Kathmandu University Medical Journal. 2005; 3(2)10:123-129.

5. K Anding, P Gross, JM Rost, et al. The influence of uraemia and haemodialysis on neutrophil phagocytosis and antimicrobial killing. Nephrol Dial Transplant. 2003;18(10):2067-2073.

6. SK Jadav, SM Sant, VN Acharya. Bacteriology of Urinary Tract Infection in Patients of Renal Failure Undergoing Dialysis. J Postgrad Med, India.1977;23(1):10-18.

7. R Funfst uck, U Ott, KG Naber. The interaction of urinary tract infection and renal insufficiency. International Journal of Antimicrobial Agents. 2006;72-77.

8. Foley RN. Infections in patients with chronic kidney disease. Infect Dis Clin North Am. 2007;21:659-672.

9. Gilbert DN. Urinary Tract infections in patients in chronic renal insufficiency. Clin J Am Soc Nephrology. 2006;1:327-331.

10. Zafer Tandogdu, Tommmaso Cai, Bela Koves, Florian Wagenlehner, Truls Erik. Urinary tract infections In immunocmpromised patients with Diabetes, Chronic Kidney Disease, and Kidney Transplantation. European Urology Focus. 2016;2:394-399.

11. Suresh Jaiswal, Rameshwar Das et al. Bacteriological Study of Urinary Tract Infection in Male Patients Undergoing Dialysis due to Chronic Kidney Disease in Tertiary Care Hospitals in Nepal. Research and Reviews: A Journal of Life Sciences. 2013;3:8-19.

12. Geerlings and Associates. Asymptomatic Bacteriuria May Be Considered a Complication in Women With Diabetes. Diabetes Care. 2000;23:744-749.

13. Raheela Mohsin, Khurram Mutahir Siddiqui. Recurrent urinary tract infections in females. Review Article. J Pak Med Assoc. 2010;60(1):55.

14. Monica -Cheesbrough District Laboratory Practice in Tropical Countries. Second edition, Part 2. 2012;107-115.

15. National Committee for Clinical Laboratory Standards (NCCLS). Performance standards for antimicrobial susceptibility testing. 16th Informational Supplement. M100-S12 NCCLS. Oxford University Press, USA. Wayne, PA. 2006.

16. Helen I McDonald, Sara L Thomas, Dorothea Nitsch. Chronic kidney disease as a risk factor for acute community-acquired infections in high-income countries: a systematic review. BMJ Open. 2014;4:e004100. doi:10.1136/bmjopen-2013-004100.

17. James MT, Laupland KB, Tonelli M et al. Risk of bloodstream infection in patients with chronic kidney disease not treated with dialysis. Arch Intern Med. 2008;168:2333-23399.

18. Getenet Beyene, Wondewosen Tsegaye . Bacterial Uropathogens in Urinary tract infection and Antibiotic Susceptibility Pattern in Jimma University Specialized Hospital, Southwest Ethiopia. Ethiop J Health Sci.2011;21(2):141-145.

19. Saitoh H, Nakamura K, Hida M. Urinary tract infection in oliguric patients with chronic renal failure. J Urol. 1985;133:990-9310 .

20. Richa C, Bhushan CS, Kumar SP, Dev PN, Nabaraj P .Bacteriology of Urinary Tract Infection of Chronic Renal Failure Patients Undergoing Hemodialysis.J Microbiol Exp. 2016;3(3):00089. 


\section{REFERENCES}

21. Shailesh C Trivedi et al. Acute Pyelonephritis in Diabetics and Non-Diabetics. Journal of Clinical and Diagnostic Research. 2016;10(10): OC26-OC2928 28 .

22. Go biewska J, D bska- lizie A, Komarnicka J, Samet A, Rutkowski B. Urinary tract infections in renal transplant recipients. 2011;43(8):2985-2990.

doi: $10.1016 /$ j.transproceed.2011.07.010

23. Kalpana Devi Venkatesan, Senthil Chander, Karthiga Loganathan, Kalavathy Victor. Study on asymptomatic bacteriuria in diabetic patients. International Journal of Contemporary Medical Research. 2017;4(2):480-483.

24. Renko et al. Meta-analysis of the significance of asymptomatic bacteriuria in diabetes.Diabetes Care. 2011;34:230-235.

25. Ragnarsdóttir B et al. Genetics of innate immunity and UTI susceptibility. Nat. Rev. Urol. 2011;8:449-468.

26. Falah S. Manhal, Ammer A. Mohammed, Kais H. Ali. Urinary tract infection in Hemodialysis patients with renal failure . J Fac Med, Baghdad. 2012;54(1):38-41.

27. Khushbu Yadav, Satyam Prakash. Antimicrobial Resistance Pattern of Uropathogens Causing Urinary Tract Infection (UTI) Among Diabetics . Biomedical Research International. (C) 2016 Jakraya Publications (P) Ltd. 2016;1:07-15.

28. Ranjana Badhan,Vijay Singh,Anureet Kaur.Evaluation Of Bacteriologicalprofile And Sensitivity Patterns With Urinary Tract Infection. Indian J Urol.2016;32(1):50-56.

29. Chih-Yen HSIAO1 et al. Urinary tract infection in patients with chronic kidney disease. Turk J Med Sci . 2014;44:145-149.

30. Dr. Edward Njogu Mainathe. Prevalence of Urinary Tract Infection In Kidney Transplant Recipients at Kenyatta National Hospital. A Dissertation Submitted In University Of Nairobi. 2014.

31. Jamal Haider JS, Hasan A, Bin-Tahir K. Frequency of Urinary Tract Bacterial Infection and their Susceptibility Patterns among Hemodialysis Patients. J Microbiol Exp. 2016, 3(3): 00093.

32. Ravindranath Gangane and Javeria Firdous.Isolation and Antibiotic Sensitivity Pattern of Extended Spectrum Beta Lactamases (ESBL) Producing Escherichia coli Isolated from Urinary Tract Infection. Int.J.Curr.Microbiol.App.Sci (2017) 6(6): 279-286. https://doi.org/10.20546/ijcmas.2017.606.034. 